\title{
MT-INSAR LANDSLIDE MONITORING WITH THE AID OF HOMOGENEOUS PIXELS FILTER
}

\author{
X. J. Liu ${ }^{1}$, C. Y. Zhao ${ }^{1,2,3 *}$, B. H. Wang ${ }^{1}$, W. F. Zhu ${ }^{1}$ \\ ${ }^{1}$ School of Geology Engineering and Geomatics, Chang'an University, Xi'an 710054, China; Xiaojie_Liu_cd@163.com (X.L.); \\ zhaochaoying@163.com (C.Z.); baohangwang@163.com (B.W.); wenfeng_zhu@163.com (W.Z.) \\ ${ }^{2}$ State Key Laboratory of Geo-Information Engineering, Xi'an, 710054, China \\ ${ }^{3}$ National administration of surveying, mapping and geo-information, Engineering research center of national geographic \\ conditions monitoring, Xi'an, 710054, China
}

\section{Commission III, WG III/3}

KEY WORDS: Goldstein filter, Homogeneous pixels filter, TerraSAR-X, SBAS, Landslide monitoring

\begin{abstract}
:
SAR interferograms are often contaminated by random noises related to temporal decorrelation, geometrical decorrelation and thermal noises, which makes the fringes obscured and greatly decreases the density of the coherent target and the accuracy of InSAR deformation results, especially for the landslide monitoring in vegetated region and in rainy season. Two different SAR interferogram filtering methods, that is Goldstein filter and homogeneous pixels filter, for one specific landslide are compared. The results show that homogeneous pixels filter is better than Goldstein one for small-scale loess landslide monitoring, which can increase the density of monitoring points. Moreover, the precision of InSAR result can reach millimeter by comparing with GPS time series measurements.
\end{abstract}

\section{INTRODUCTION}

Synthetic aperture radar interferometry (InSAR), a highly visual technique, has been widely used in landslide detection and monitoring in recent decades (Zhao et al., 2012; Bayer et al., 2017). Generally, obtaining accurate phase differences with two or more SAR images is essential for the precise landslide detection and monitoring with InSAR technique. As SAR interferogram is always contaminated by heavy noises, and several interferogram filtering methods have been proposed to decrease them. Currently, two main filtering categories including spatial domain filtering techniques and frequency domain filtering techniques are commonly used. The former one includes mean and median filter (Lanari et al., 1996; Candeias et al.,1995), and Lee filter (Lee 1983a). These methods can reduce noises at the expense of spatial resolution. The fringe structure is often obscured especially in dense-fringe areas. In order to keep the spatial resolution and reduce noises, some improved filtering approaches have been proposed, which includes slope-compensated mean filter based on local frequency estimation (Suo et al., 2010), window-direction or size-dependent adaptive filter (Lee at al., 1998) and nonlocal filter (Parrilli et al., 2012; Deledalle et al., 2015). The core idea of the later one is to transform interferograms from the spatial domain to the frequency domain and then smooth high frequency signals to reduce noises. The most typical ones are Goldstein filter algorithm (Goldstein and Werner 1998) and its improved versions (Baran et al., 2003; Li et al., 2008; Sun et al.,
2009; Zhao et al., 2012; Jiang et al., 2014; Song et al., 2015; Zhao, Jiang, and $\mathrm{He} 2016$ ). The Goldstein filter and its improved versions have notable smoothing capability for most cases. However, in some low coherence regions, the abovementioned algorithms can hardly reserve dense effective targets to obtain useful surface deformation.

Dangchuan landslide group (Figure 1), located in the arid loess plateau in northwestern China, has been suffering a large number of loess landslides in the past tens of years due to massive agricultural irrigation (Qi et al., 2017). On the other hand, this region can hardly get dense InSAR monitoring results due to the heavy decorrelation caused by rainfall and vegetation coverage especially from June to September. In order to increase the density of monitoring points, homogeneous pixels filter (phase filtering based on coherence magnitude as a weight and homogenous pixels for each reference pixel) is used to reduce the phase noises. Firstly, statistically homogeneous pixels for each reference pixel are determined under the assumption that similar surface targets have the similar backscattering properties. Then the intensity and coherence are accurately estimated by using two robust estimators of updated Lee intensity filter algorithm (Jiang et al., 2017) and non-parameter Bootstrap algorithm (Jiang et al., 2014) based on identified statistically homogeneous pixels. Finally, the interferometric phase is filtered through homogeneous pixels filter before the SBAS InSAR processing.

\footnotetext{
* Corresponding author
} 

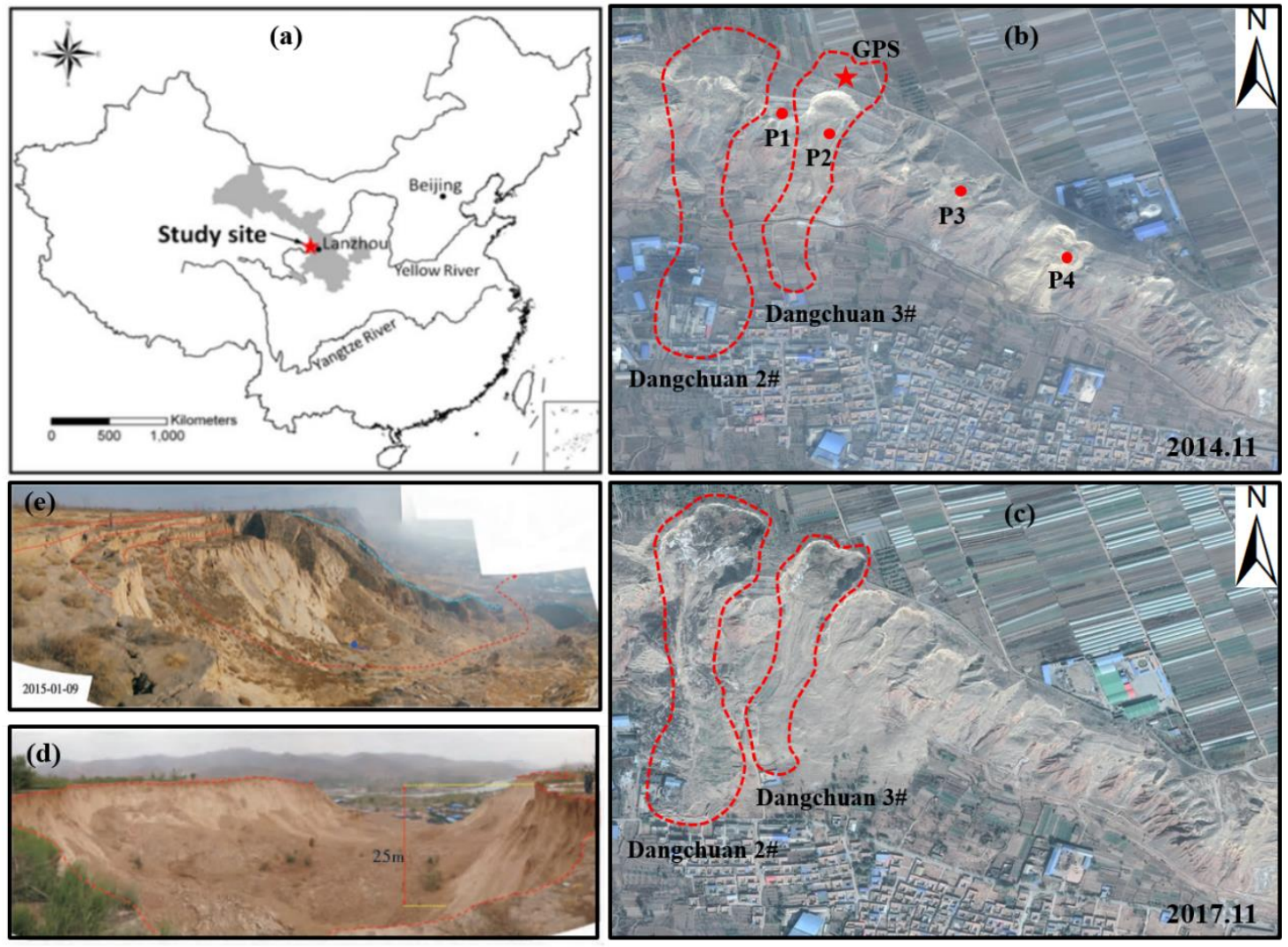

Figure 1. (a) Location of study region; (b) remote sensing image of Dangchuan landslide group in November 2014 (The red pentagram is the location of GPS, and points P1 to P4 will be analyzed in Figure 3, the red dotted line is the boundary of landslides.); (c) remote sensing image of the landslide group in November 2017; (d) the photo after Dangchuan No. 2 landslide occurred; (e) the photo before Dangchuan No. 2 landslide occurred. (Red lines indicate the fractures before the landslide occurred, and the red dotted line shows the sliding direction (revised after Xu et al., 2016).)

\section{METHODLOGY}

\subsection{Fast statistically homogeneous pixel selection algorithm}

The core of fast statistically homogeneous pixels selection is to construct the confidence interval for each pixel by applying the central limit theorem, and then determines homogeneous pixels using this interval (Jiang et al., 2015; Jiang et al., 2016). For an generic point $p$, assuming its $N$ temporal intensity samples are $\left\{A_{1}, A_{2}, \cdots, A_{N}\right\}$, the point estimate of mean $\mu(p)$ can be given as,

$$
\bar{A}(p)=\left(A_{1}(p)+A_{2}(p)+\cdots+A_{N}(p)\right) / N
$$

According to the central limit theorem, $\bar{A}(p)$ is gradually close to Gaussian distribution with the increase of samples number $N$. Therefore, the interval estimates of $\bar{A}(p)$ can be acquired with the following form,

$$
\begin{aligned}
& P\left\{\mu(p)-Z_{1-\alpha / 2} \sqrt{\operatorname{Var}(A(P)) / N}<\bar{A}(p)<\mu(p)+Z_{1-\alpha / 2}\right. \\
& \cdot \sqrt{\operatorname{Var}(A(p)) / N}\}=1-\alpha
\end{aligned}
$$

where $Z_{1-\alpha / 2}$ is the $1-\alpha / 2$ percentile of the standard normal probability density function, and $\operatorname{Var}(A(p))$ is true variance of point $p$. It should be noted that the variation coefficient of single look intensity images $C V=\sqrt{\operatorname{Var}(\bullet)} / \mu(p) \approx 0.52$ in homogeneous regions (Jiang et al., 2016). Therefore, we can get

$$
\begin{aligned}
& P\left\{\mu(p)-Z_{1-\alpha / 2} \bullet 0.52 \bullet \mu(p) / \sqrt{N}<\bar{A}(p)<\mu(p)+Z_{1-\alpha / 2} \bullet 0.52\right. \\
& \text { • } \mu(p) / \sqrt{N}\}=1-\alpha
\end{aligned}
$$

Formula (3) is a certain interval in case of $\mu(p)$ is known. Consequently, when choosing statistically homogeneous pixels for reference pixel $p_{k}$ in real processing, we first use $\bar{A}_{p k}$ as the true value of $p_{k}$, i.e., $\mu(p k) \approx \bar{A}(p k)$. Then $K-1$ ( $K$ is the number of tested pixel) neighbors being tested in a fixed window are checked one by one by using formula (3) and their sample mean $\bar{A}(p i), i=1, \cdots k-1, k+1, \cdots, K$. All pixels are preliminarily regarded as homogeneous pixel, if their values lie in this interval regardless of the connectivity of pixels. Because of the small SAR data number $N$, the initial estimate of mean $\mu(p k)$ is unreliable and the estimation will be iteratively processed by averaging all pixels in $\Omega$ init . After a refined set $\Omega$ for $p_{k}$ is achieved, formula (3) is used again for the same neighbors. It should be noted that $\alpha=50 \%$ and $\alpha=5 \%$ are used to determine $\Omega$ init and $\Omega$ in the processing, respectively.

\subsection{Unbiased coherence estimation based on non-parameter Bootstrap algorithm}

The spatial coherence of a generic interferometric pair can be estimated as follows. 


$$
\gamma=\frac{\sum_{n=1}^{N}\left|y_{1}^{(n)} y_{2}^{*(n)}\right|}{\sqrt{\sum_{n=1}^{N}\left|y_{1}^{(n)}\right|^{2} \sum_{n=1}^{N}\left|y_{2}^{(n)}\right|^{2}}}
$$

where $y_{1}^{(\bullet)}$ and $y_{1}^{(\bullet)}$ are two single look complex (SLC) images, $N$ is the number of homogeneous pixels in a fixed window. In order to obtain the accurate estimate of coherence, three kinds of error sources must be removed: the non-stationarity of radar signals, the phase gradient and the biased estimator (Jiang et al., 2014). The non-stationarity of signals can be removed by adopting homogeneous pixels. The phase gradient is removed by the method of topography phase compensation interferometric fringe. Finally, the bias of estimator is removed by non-parameter Bootstrap method and the unbiased coherence is acquired.

In the case of coherence estimation, each pixel in the fixed window consists of a pair of complex values $x_{i}=\left(y_{1 i}, y_{2 i}\right)$, $i=1, \cdots, N$. Therefore, all pixels in the fixed window can be written as $X=\left(x_{1}, x_{2}, \cdots, x_{N}\right)$, and every element of $X$ is independent and identically distributed. A Bootstrap sample $X^{*}=\left(x_{1}^{*}, x_{2}^{*}, \cdots, x_{N}^{*}\right)$ is built by randomly sampling from the original observations $\left(x_{1}, x_{2}, \cdots, x_{N}\right) \quad N$ times with replacement. We can obtain a group of samples $X_{1}^{*}, X_{2}^{*}, \cdots, X_{R}^{*}$ through repeated sampling with $R$ times. Bootstrap replications $\gamma_{1}^{*}, \gamma_{2}^{*}, \cdots, \gamma_{R}^{*}$ are acquired by calculating the coherence value of $\gamma^{*}$ for each a group of samples using equation (4). Therefore, unbiased estimate $\hat{\gamma}$ is computed approximatively by correcting the bias between these replicate estimate $\gamma^{*}$ and original estimate $\gamma$.

$$
\hat{\gamma}=2 \gamma-\frac{1}{R} \sum_{i=1}^{R} \gamma_{i}^{*}
$$

\section{EXPERIMENT}

19 scenes of X-band Spot mode TerraSAR-X data from January 2016 to November 2017 are involved to monitor the deformation of Dangchuan landslide group, and the pixels spacing in the azimuth and range are 0.9 and 1.26 meter, respectively. The temporal baseline and the spatial baseline thresholds are set to 60 days and $250 \mathrm{~m}$ to generate high quality interferometric pairs, and the topographic phase is removed by three meters resolution unmanned aerial vehicle (UAV) DEM. Then two filtering schemes are designed including Goldstein filter and homogeneous pixels filter. For the first scheme, multi-looking number two in both range and azimuth directions is set to increase the signal-to-noise ratio (SNR) and the filtering is operated twice. While for the second scheme, only single look interferograms are generated to preserve the phase signatures of natural structures. Followed by the amplitude-based adaptive multi-looking of InSAR stacks (Parizzi et al., 2011). The previous determined homogeneous pixels are averaged by statistical tests and stochastic model (Jiang et al., 2015). Due to the landslide frequent occurrences in this area, DEM error cannot be neglected. Therefore, the residual topographic phase will be removed. Lastly, time series deformation results will be got with small baseline subsets (SBAS) InSAR technique. Figures 2(a) and 2(b) show the average deformation rate maps of Dangchuan landslide group with the aid of Goldstein filter and homogeneous pixels filter, respectively. The second method can give much more coherent targets, even in the low coherence regions, which will be greatly beneficial to the spatial landslide analysis. Figure 3 shows the time series deformation comparison for point P1 to P4 between Goldstein and homogeneous pixels filter methods. Both two methods can give consistent results in point P1, P3 and P4. While the difference between two methods is $23.5 \mathrm{~mm}$ in point $\mathrm{P} 2$, which may be caused by the unwrapping error in point $\mathrm{P} 2$ due to the large deformation gradient and sparse coherent targets. In Figure 4, the results are validated with time series GPS measurements, which shows that the standard deviation of the Goldstein filter and homogeneous pixels filter results are approximately $2 \mathrm{~mm}$ and $4.4 \mathrm{~mm}$, respectively.

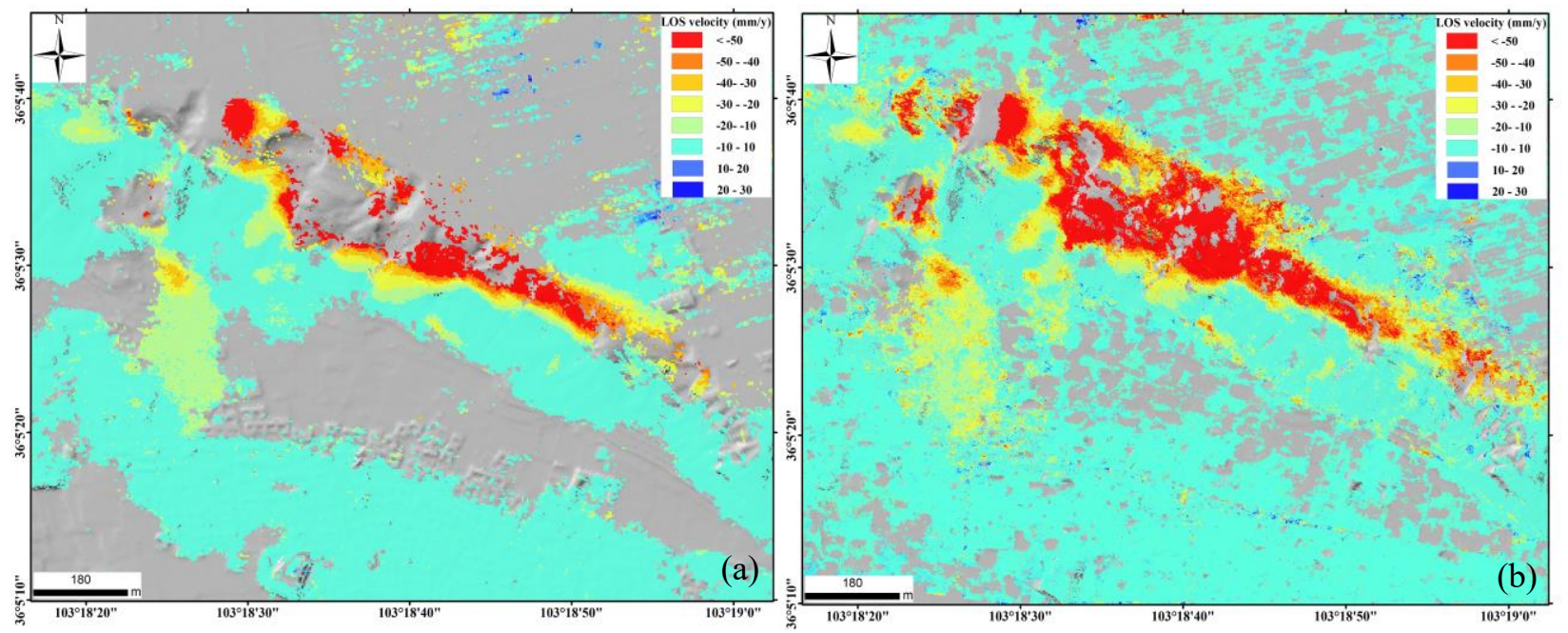

Figure 2. Average deformation rate map of Dangchuan landslide group. (a) Calculated with Goldstein filter; (b) Calculated with homogeneous pixels filter. 

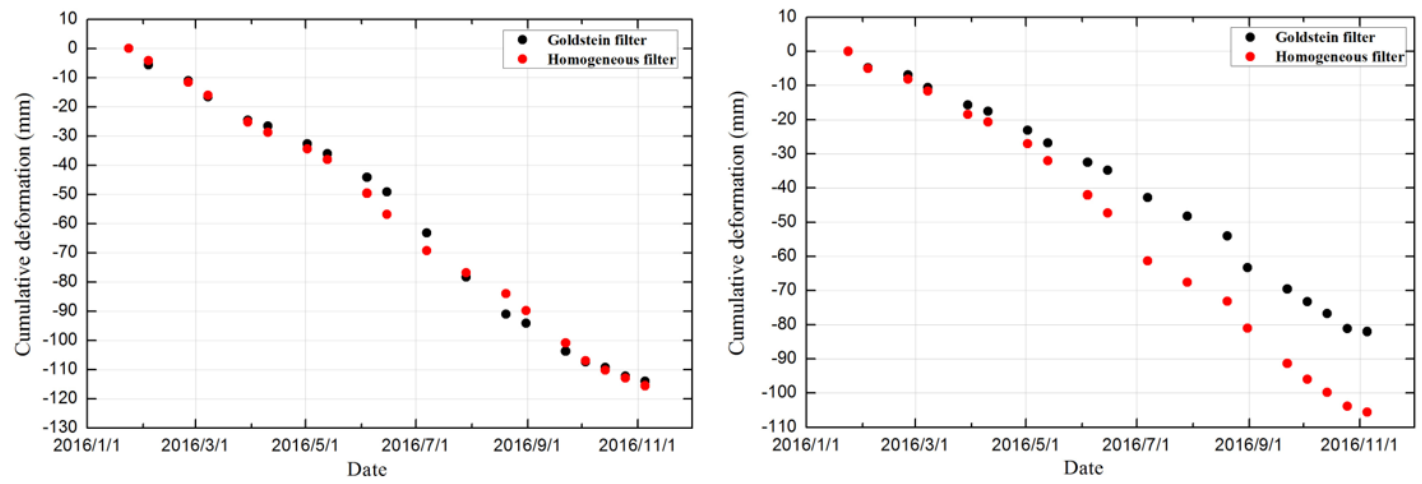

P1

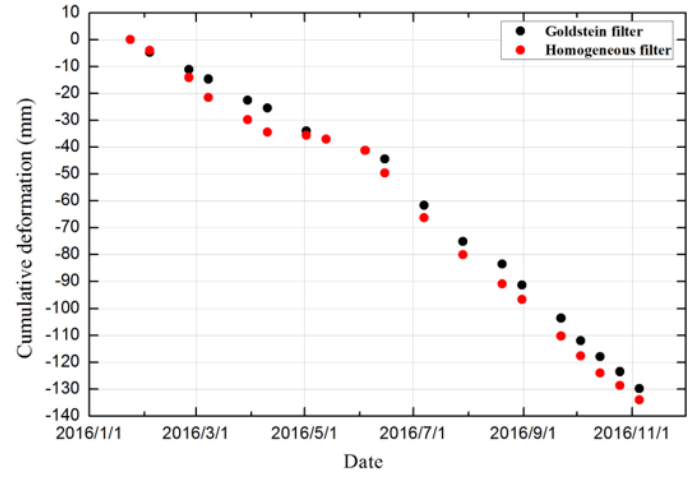

P3

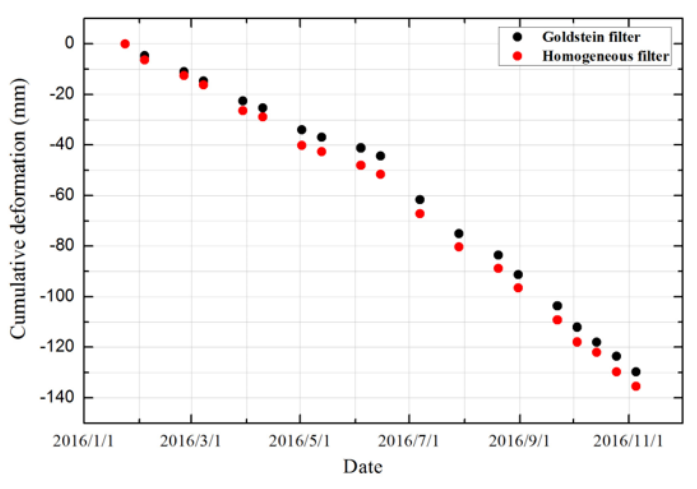

P4

Figure 3. Time series deformation comparison for points P1 to P4 in Figure 1(b) between Goldstein and homogeneous pixels filter methods.

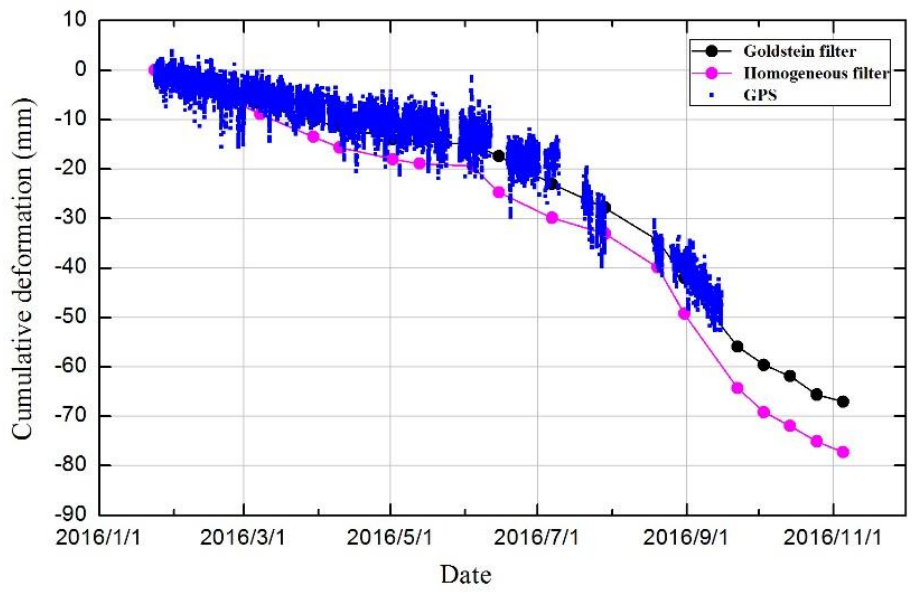

Figure 4. Comparison of two filtering methods results with GPS measurements

\section{CONCLUSIONS}

In this study, the method of homogeneous pixels filter is tested to monitor the deformation of Dangchuan landslide group. Although the precision of homogeneous pixels filter result is slightly lower than the one of Goldstein filter, it can increase the density of monitoring points significantly, which can provide detailed landslide information.

\section{ACKNOWLEDGEMENTS}

This research was funded by National Program on Key Basic Research Project (973 Program) (Grant No. 2014CB744703) and Natural Science Foundation of China projects (NSFC)
(Grant No. 41731066 and 41628401). TerraSAR data are provided by DLR under grant GEO3039.

\section{REFERENCES}

Baran, I., Stewart, M. P., Kampes, B.M., Perski, Z., 2003. A modification to the Goldstein radar interferogram filter. IEEE Transactions on Geoscience and Remote Sensing, 41(9), pp. 2114-2118.

Bayer, B., Simoni, A., Schmidt, D., Bertello, L., 2017. Using advanced InSAR techniques to monitor landslide deformations induced by tunneling in the Northern Apennines, Italy. Engineering Geology, 226, pp. 20-32. 
Candeias, A. L. B.; Mura, J. C.; Dutra, L. V.; Moreira, J. R. (1995): Interferogram phase noise reduction using morphological and modified median filters: Geoscience and Remote Sensing Symposium, 1995. IGARSS'95. 'Quantitative Remote Sensing for Science and Applications', International IEEE, Vol.1, pp. 166-168.

Deledalle, C., Denis, L., Tupin, F., Reigber, A., Jager, M., 2015. NL-SAR: A unified nonlocal framework for resolution-preserving (Pol) (In) SAR denoising. IEEE Transactions on Geoscience and Remote Sensing, 53(4), pp. 2021-2038.

Goldstein, R., Werner, C., 1998. Radar interferogram filtering for geophysical applications. Geophysical Research Letters, 25(12), pp. 4035-4038

Qi, X., Xu, Q., Liu, F. Z., 2017. Analysis of retrogress loess flowslides in Heifangtai, China. Engineering Geology, pp. $1-10$.

Jiang, M., Ding X. L., Hanssen, F., Chang, L., 2015. Fast statistically homogeneous pixel selection for covariance matrix estimation for multitemporal InSAR. Transaction on geoscience and remote sensing, 53(3), pp.1213-1224.

Jiang, M., Miao, Z. M., Gamba, P., 2017. Application of Multitemporal InSAR Covariance and information fusion to robust road extraction. Transaction on geoscience and remote sensing, 55(6), pp. 3611-3621.

Jiang, M., Ding, X. L., Tian, X., Malhotra, R., Kong, W., 2014. A hybrid method for optimization of the adaptive Goldstein filter. ISPRS Journal of Photogrammetry and Remote Sensing, 98, pp. 29-43.

Jiang, M., Ding, X. L., He, X. F., Li, Z.W., Shi, G.Q., 2016. FaSHPS-InSAR technique for distributed scatterers: A case study over the lost hills oil field, California. Chinese J. Geophys., 59(10), pp. 3592-3603. (in Chinese)

Jiang, M., Ding, X. L., Li, Z. W., 2014. Hybrid approach for unbiased coherence estimation for multitemporal InSAR. IEEE Transaction on Geoscience and Remote Sensing, 52(5), pp. 2459-2473.

Lanari, R., Fornaro, D., Riccio, D., Migliaccio, M., 1996. Generation of digital elevation models by using Sir-C/X-SAR multifrequency two-pass interferometry: the Etna case study. IEEE Transactions on Geoscience and Remote Sensing, 34, pp. 1097-1114.

Lee, J. S., 1983a. Digital image smoothing and the sigma filter. Computer Vision, Graphics, and Image Processing, 24(2), pp. 255-269.

Lee, J. S., Papathanassiou, K. P., Ainsworth, T. L., Grunes, M. R., Reigber, A., 1998. A New Technique for Noise Filtering of SAR Interferometric Phase Images. IEEE Transactions on Geoscience and Remote Sensing, 36 (5), pp. 1456-1465.

Li, Z. W., Ding, X. L., Huang, C., Zhu, J. J., Chen, Y. L., 2008. Improved filtering parameter determination for the Goldstein radar interferogram filter. ISPRS of Photogrammetry and Remote Sensing, 61(6), pp. 621-634.

Parizzi, A., Ramon, B., 2011. Adaptive InSAR Stack Multilooking Exploiting Amplitude statistics: a comparison between different techniques and practical results. Remote sensing letters, 8(3), pp. 441-445.

Parrilli, S., Poderico, M., Angelino, C., Verdoliva, L., 2012. A nonlocal SAR image denoising algorithm on LLMMSE wavelet shrinkage. IEEE Transactions on Geoscience and Remote Sensing, 50 (2), pp. 606-616.

Song, R., Guo, H. D., Liu, G., Perski, Z., Yue, H. Y., Han, C. M., Fan, J. H., 2015. Improved Goldstein SAR interferogram filter based on adaptive-Neighborhood technique. IEEE Geoscience and Remote Sensing Letters, 12(1), pp. 140-144.

Sun, Q., Zhu, J. J., Li, Z. W., Yin, H. J., Hu, B., Jiang, M., 2009. A new adaptive InSAR interferogram filter based on SNR. Acta Geodaetica et Cartograohica Sinica, 6(10). pp. 1024-1028. (in Chinese)

Suo, Z., Li, Z., Bao, Z., 2010. A new strategy to estimate local fringe frequencies for InSAR phase noise reduction. IEEE Geoscience and Remote Sensing Letters, 7(4), pp. 771-775.

Xu, Q., Peng, D. L., Qi, X., Dong, X. J., Li, H. J., Ju, Y. Z., 2016. Dangchuan 2\# landslide of April 29, 2015 in Heifangtai area of Gansu province: characteristics and failure mechanism. Journal of Engineering Geology, 24(2), pp. 167-180. (in Chinese)

Zhao, C. Y., Zhang, Q., He, Y., Peng, J. B., Yang, C. S., Kang, Y., 2016. Small-scale loess landslide monitoring with small baseline subsets interferometric synthetic aperture radar technique-case study of Xingyuan landslide, Shaanxi, China. Journal of applied remote sensing,10(2), pp. 1-14.

Zhao, C. Y., Zhang, Q., Zhang, J., 2011. Deformation monitoring of ground fissure with SAR interferometry in Qing Xu. Journal Engineering Geology, 19(1), pp. 70-75.

Zhao, C.Y., Lu, Z., Zhang, Q., Fuente, J. D. L., 2012. Large-area landslide detection and monitoring with ALOS/PALSAR imagery data over Northern California and Southern Oregon, USA. Remote sensing of Environment, 124(9), pp. 348-359.

Zhao, W. S., Jiang, M., He, X. F., 2016. Improved adaptive Goldstein interferogram filter based on second kind statistics. Acta Geodaetica et Cartographica Sinica, 45(10), pp. 1200-1209. (in Chinese) 